\title{
POLYPROPYLENE TENSILE TEST UNDER DYNAMIC LOADING
}

\author{
Mahomet Khlif, N. Masmoudi, Ch. Bradai \\ National School of Engineers of Sfax, University of Sfax \\ Analysis Laboratory of Electro Mechanical Systems \\ BP.1173. W. 3038 Sfax, Tunisia \\ fax: + 21674275595 \\ e-mail:khlifmohamed@yahoo.fr
}

\begin{abstract}
In this paper, a testing method is developed to determine the dynamic stress-strain responses of the polypropylene $(P P)$ under high strain rate uniaxial tension conditions. The main objective of this study is to determine the dynamical behaviour of PP materials under transitory loading. To this effect, an experimental machine using a mixed technique of Hopkinson tension bar and a sensing block system is developed in order to study the dynamic tensile stress-strain behaviour of the low-strength and low mechanical impedance specimen. A pendulum pulse technique is employed in dynamic tension experiments to ensure that valid experimental conditions are satisfied. Results show that, compared with quasi-static behaviour, dynamic tension causes smaller failure strains.

Among other, the experimental set-up, mechanical characteristic, dynamic tensile specimen, quasi-static tensile test: stress-strain curve for $0.8 \mathrm{~s}-1$ strain rate, the incident and the transmitted waves recorded by the stain gauges located on the bar and the sensing block under a dynamic tensile test, the axial strain on the specimen versus time under a dynamic tensile test, the axial stress on the specimen versus time under a dynamic tensile test, dynamic tensile test: stress-strain curves of the PPC7712 polymer for various strain rates, the specimens after rupture quasi-static test and dynamic test are presented in the paper.
\end{abstract}

Keywords: Dynamic tension, Polypropylene, High strain rate, Hopkinson bar, Sensing block

\section{Introduction}

Polymeric materials have been used widely in engineering applications due to their low production cost and light weight. Polymeric materials are subjected to dynamic loading and highstrain-rate deformation in a variety of important applications such as aircraft and automotive components, as well as during high-speed processes such as extrusion and blow moulding. However, the dynamic mechanical responses of polymeric materials under transitory loading are not completely understood. In particular, it is unclear whether the dynamic compressive behaviour would be a reasonable reference for the corresponding tensile behaviour since the differences between dynamic tensile and compressive responses have rarely been addressed. The study of materials properties under quasistatic to dynamic loading depends on the strain rates range. The strain rate for dynamic domain begins at approximately $10 \mathrm{~s}^{-1}$ (Hamoda 1998) [1].

Polymeric responses under impact have been studied since Kolsky's (1949), [2] pioneering work. For example, (Q. M. L et al., 2009), [3] used a split Hopkinson pressure bar (SHPB) to determine the dynamic compressive stress-strain behaviour of Lucite and Micarta. (Chou et al., 1973), [4] measured the dynamic compressive behaviour of plastics using the same method. (Walley et al., 1989), [5] used high-speed photography to determine the rapid deformation behaviour of a range of polymers in compression. (Dioh et al., 1993), [6] noticed that specimen thickness was an important parameter when using the SHPB to test polymers. A few studies of polymeric material dynamic tensile behaviour are found in the literature. In this study, the dynamic response of a heterophasic copolymer polypropylene PPC 7712 under a dynamic uniaxial tensile loading condition has been determined experimentally. In these experiments, a conventional Hopkinson bar was modified and a sensing block is used in order to measure the weak signals transmitted from the specimens of low strength and low mechanical impedance. A pendulum was 
applied to control the incident pulses in a tensile experiment in order to achieve dynamic stress equilibrium and homogeneous deformation in the specimen. A conventional tensile machine was used to conduct the quasi-static behaviour of PP materials.

In the following sections, the experiments are described and the results are presented with the appropriate discussions.

\section{Experimental set-up}

Experimental machine used in this study include a Hopkinson tension bar modified to conduct dynamic tension experiments on polymer specimens and a sensing block to perform dynamic tensile tests (Khlif et al 2008) [7]. A conventional tensile machine is used for quasi-static experiments.

\subsection{Description of the dynamic loading machine}

The dynamic loading machine presented in Fig. 1 is composed of three parts (Khlif et al 2008), [7]. The first part represents the shock generating system used to excite the input bar. It includes a rotating pendulum that impacts the end part of the input bar. The intensity of the pendulum shock can be changed using a range of variable masses. The speed of the excitation impact is a function of the pendulum altitude position. To commands the shock generating system and allows only one impact, a pneumatic device is implemented.

The shock receiving system represents the second part; it is illustrated in Fig. 1. It is composed of an input bar which receives the shock excitation generated by the pendulum and transmits the corresponding strain wave to the specimen located between the input bar and the sensing block. The geometrical sizes of the incident bar and the sensing block as well as the corresponding materials are presented in the following sections. The input bar is instrumented in order to measure the force. The choice of a long bar enables us to solve the waves superposition problem (incident and reflected waves) and to measure the loading force for a dynamic test. Moreover, the transmitting incident wave from the bar is transmitted to the specimen. This wave is finally received by the sensing block, which is composed, of a sensing receiver and a base block. A strain gage located at the sensing receiver measures the corresponding transmitted stress wave. Due to the important relative large mass of the base block, the stress wave moving from the sensing receiver is dissipated after some reflections between the base block upper surfaces.

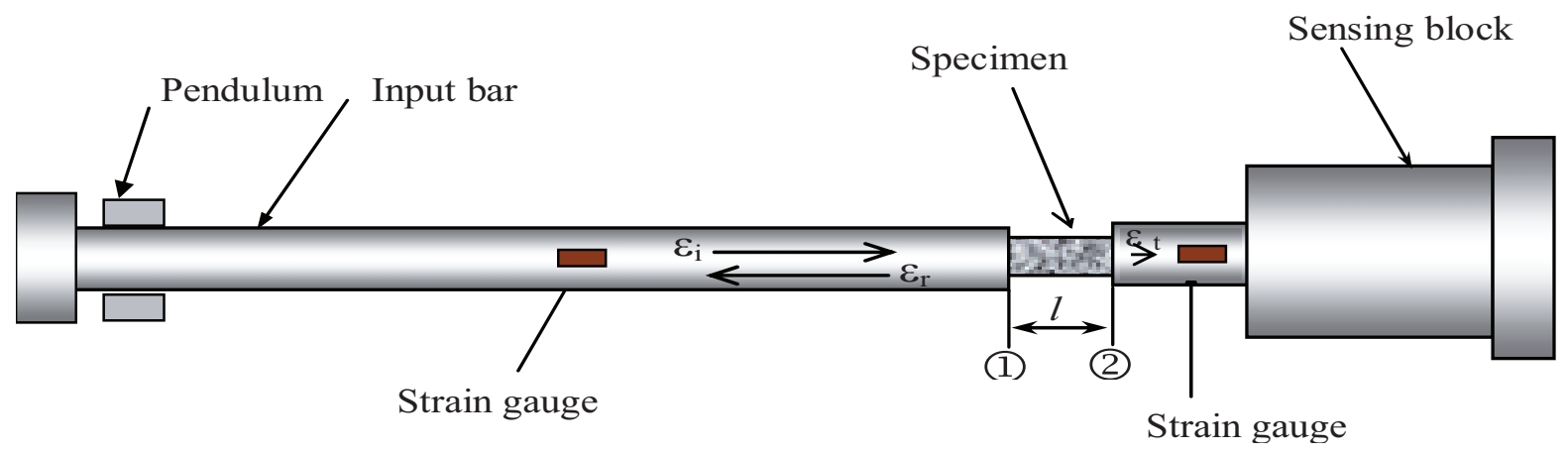

Fig. 1. The experimental set-up

The technique described above has many advantages compared with the Hopkinson bar system: The important mass of the base block allows a negligible reflection waves disturbance and the short length of the sensing receiver allows a long measuring time.

The mechanical characteristics (Young' modulus, Poisson's ratio, and density) of the investigated polymer, the bar and the sensing block are presented in Tab. 1. 
Tab. 1. Mechanical characteristic of polymer PPC7712, the bar and the sensing block

\begin{tabular}{|c|c|c|c|c|c|}
\cline { 2 - 5 } \multicolumn{1}{c|}{} & Materiel & $\rho$ & $\mathrm{E}$ & $v$ & $\mathrm{Re}$ \\
\hline specimen & polypropylene & $1400 \mathrm{~kg} / \mathrm{m}^{3}$ & $1.4 \mathrm{GPa}$ & 0.4 & $9 \mathrm{MPa}$ \\
\hline $\begin{array}{c}\text { bar and sensing } \\
\text { block }\end{array}$ & aluminium & $2830 \mathrm{~kg} / \mathrm{m}^{3}$ & $71 \mathrm{GPa}$ & 0.34 & $41 \mathrm{MPa}$ \\
\hline
\end{tabular}

The last part of the dynamic loading machine represents the data acquisition system which analyses the signals (incident $\varepsilon_{i}$, transmitted $\varepsilon_{t}$ and reflected $\varepsilon_{r}$ waves) measured by two pairs strain gauges type Kyowa KFG-5-120-C1-11. The strain gauges grill length is $5 \mathrm{~mm}$. The first pair is located in the middle of the input bar, the second pair is mounted on the sensing block. A data acquisition card "NI-PCI-6250" is used to collect signals issued from the gauges, which were previously amplified. The visualization and analysis of results are done using Lab-view software.

\subsection{Wave propagation analysis}

Assuming a homogeneous deformation in the specimen and identical incident and transmitted waves, the analysis based on one-dimensional wave theory (Khlif et al 2008), [7] showed the nominal strain $\varepsilon_{s}(t)$, in the specimen to be:

$$
\varepsilon_{s}(t)=\frac{C_{0}}{l} \int_{0}^{t}\left(\varepsilon_{i}-\varepsilon_{r}\right) d t
$$

where,

$l$ is the initial length of the specimen,

$\varepsilon_{i}(t)$ and $\varepsilon_{r}(t)$ are the incident and reflected strain waves in the incident bar,

$C_{0}$ is the wave celerity of the bar material.

On the other hand, the forces at the ends of the specimen are obtained by the following equations:

$$
\begin{gathered}
F_{1}=E A\left(\varepsilon_{i}+\varepsilon_{r}\right), \\
F_{2}=E A \varepsilon_{t},
\end{gathered}
$$

where,

$A \quad-$ the cross-sectional area of the bar,

$\varepsilon_{t}(t)$ - the transmitted strain waves in the sensing block of Young's modulus $E$.

Assuming equal forces at both ends of the specimen $(F 1=F 2)$ and using equations $(2 \mathrm{a})$ and (2b) yields to:

$$
\varepsilon_{t}=\varepsilon_{i}+\varepsilon_{r}
$$

Integration of equation (1) with respect to time gives the time-resolved axial strain of the specimen. The nominal axial stress $\sigma_{\mathrm{s}}$, in the specimen is determined using the following equation (Khlif et al., 2008) [7]:

$$
\sigma_{s}(t)=E \frac{A}{A_{s}} \varepsilon_{t}(t),
$$

where $A_{s}$ is the cross-sectional area of the specimen.

The sensing block system has been an effective tool for investigating the dynamic flow behaviour of ductile metals (Tanimura 2001), [8]. However, when the specimen has a low impedance, low strength material, the limitations of the split Hopkinson bars should be recognized and remedied. First, the low mechanical impedance of the specimen allows the incident bar-specimen interface to move almost freely under stress-wave loading because most 
of the incident pulse is reflected back into the incident bar. Only a small portion of the loading pulse is transmitted through the specimen into transmission bar, so the transmitted strain signal $\varepsilon_{t}(t)$ has very small amplitude. To solve this problems and to increase the magnitude of a weak transmitted signal we used a sensing block system in aluminium alloy (Fig. 1a). This method is alike to the sensing projection for impact experiments (Grolleau et al 2005), [9]. It is apparent from equation (3) that to increase the transmitted strain $\varepsilon_{t}(t)$ under the same specimen, it is necessary to reduce either the Young's modulus of the bar material, $E$, or the cross-sectional area ratio, $A / A_{s}$, or both. Both the lower Young's modulus of the aluminium alloy and a smaller cross-sectional area of the sensing block contribute to increase in magnitude of the transmitted $\operatorname{signal} \varepsilon_{t}(t)$.

At the interface, the fixing of the specimen at the end of the incident bar and the sensing block is performed by three screws. To conserving same cross-sectional area a groove is made at the end of the bar and the sensing block

As pointed out by (Chen et al. 2000), [10], in order to facilitate dynamic stress equilibrium and constant strain rate in a low-impedance specimen, a pulse shaper must be used. The sensing block undergoes the loading signal from the specimen; the latter will be in dynamic equilibrium. This technique was also used by (Tanimura 2001), [8] to determine the dynamic impact strength of mild steels.

To ensure homogeneous deformation of the specimen before fracture occurs, a pulseshaping technique was applied. Pulse shaping is an experimental technique to ensure stress equilibrium and constant strain rate in the specimen in dynamic tensile testing (Frew et al 2001), [11]. The rise time of the loading pulse was designed to be longer than the stressequilibrating time in the tensile specimen. The bar wave speed of the polypropylene is about $2 \mathrm{~km} / \mathrm{s}$. The gage length of the dynamic tensile specimens is $10 \mathrm{~mm}$. This wave takes $25 \mu \mathrm{s}$ to reach dynamic stress equilibrium in the gage section. Control of the pulse shape was achieved by launching a pendulum at the end of incident bar. The pendulum material was chosen according to the intensity loading proposed. The gradually increasing disk area upon impact by the striker allows more and more momentum to transfer from the striker to the incident bar, which significantly increases the rise time of the incident pulse. Proper choice of the pulseshaper material and dimensions controls the profile of the incident pulse. The amplitude and duration of an incident pulse are controlled by varying the pendulum velocity and weight. In dynamic tensile experiments with pulse shaping, the specimen fractured at two places in a short $(10 \mathrm{~mm})$ gage section, as shown in (Fig. 2) which is an indication that dynamic stress equilibrium had been reached before failure occurred.

\subsection{Materials and specimens}

A heterophasic multi-phase copolymer polypropylene (named PPC7712) supplied by TotalPetrochemicals was analysed in this study. Quasi-static loading tests (simple tensile, cyclic, relaxation and torsion tests) had previously been carried out on this grade of polymer (Zrida et al 2009), [12]. PPC7712 combines good fluidity and mechanical properties. It is characterised by excellent impact resistance and makes for faster cycling because it lends itself to early remoulding. Its melt flow index is $13 \mathrm{~g} / 10 \mathrm{~min}$. The rate of crystallinity had previously been measured by Differential Scanning Calorimetry (DSC) using the enthalpy of fusion of $209 \mathrm{~J} \mathrm{~g} \mathrm{~g}^{-1}$ in $100 \%$ crystalline polypropylene (Krevelen 2009), [13].

The specimens were moulded by injection machine with controlled parameters. Fig. 2 illustrates the injection mould and the dimensions for the specimens used in dynamic tension tests (The standard NF ISO 8256) (Krawczak 1997), [14]. The quasi-static tension specimen is determined by the standard ISO 6200, the gage section length is $55 \mathrm{~mm}$. 

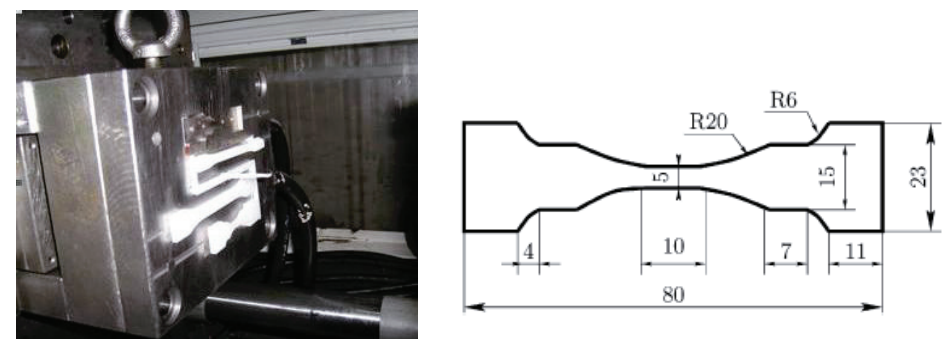

Fig. 2. Dynamic tensile specimen (thickness $5 \mathrm{~mm}$ )

\section{Experimental results}

The experiments were conducted in the mechanical department at the University of Sfax. For the dynamic tensile machine (Fig. 1), the length of the incident bar is $3000 \mathrm{~m}$. The sensing block system, manufactured of aluminium alloy, is composed of a specific sensing receiver with $15 \mathrm{~mm}$ diameter and $30 \mathrm{~mm}$ length and a base block with $100 \mathrm{~mm}$ diameter and $200 \mathrm{~mm}$ length. This important difference between the two diameters provokes the wave-trapping phenomenon.

In this study, uniaxial tension experiments on the PPC 7712 were performed using the Static Tensile Machine (MT'S) and the dynamic tensile machine described in Fig. 1, is used for four strain rates $10^{2} \mathrm{~s}^{-1}, 2.10^{2} \mathrm{~s}^{-1}, 3.10^{2}, 4.10^{2} \mathrm{~s}^{-1}$ and $5.10^{2} \mathrm{~s}^{-1}$. The time-resolved engineering stress and engineering strain profiles were recorded during the quasi-static experiments on the MTS. For the quasi-static experiments, the MT'S machine parameters are controlled in order to get a constant strain-rate in the specimen. Fig. 3 presents the evolution of the stress related to the strain of the polypropylene PPC 7712 for $0.8 \mathrm{~s}^{-1}$ strain rate. It is clear from the experiment that the PPC 7712 has elasto-plastic behaviour. For small strain $(0-0.7 \%)$, the material has a linear behaviour.

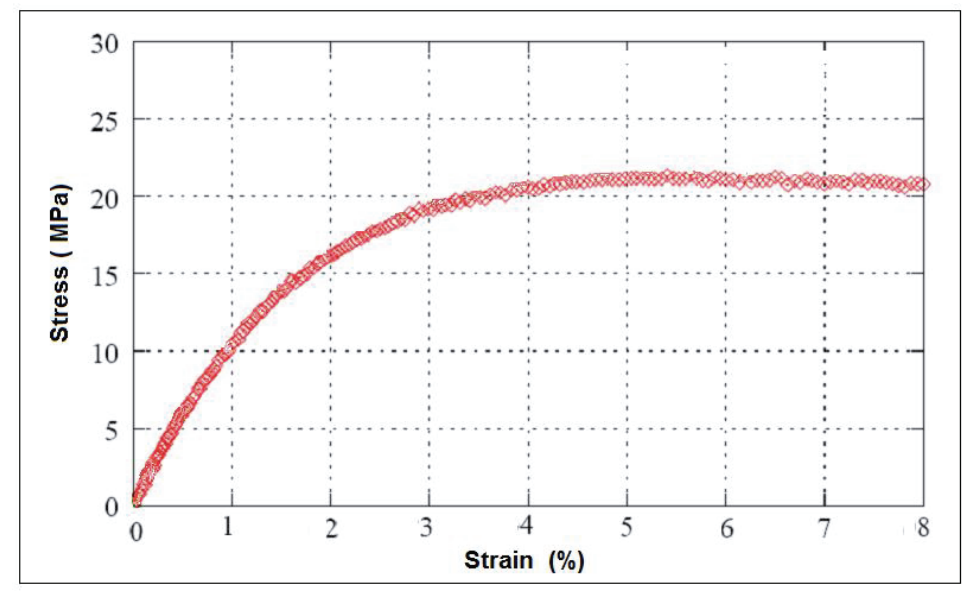

Fig. 3. Quasi-static tensile test: stress-strain curve of PPC7712 for 0.8 s-1 strain rate

During dynamic tension experiments, the incident, reflected and transmitted strain signals were recorded. Using these signals and Eqs (1) and (3), the strain, and dynamic tensile stress in the specimen are calculated.

Pulse shaping was used to achieve constant strain rates in dynamic experiments, which took a significant amount of experimental effort to find a suitable pulse shaper for a particular set of test conditions. (Fig. 5) shows a typical oscilloscope record of the dynamic tensile experiment on PPC7712 using the dynamic tensile machine. As indicated by the transmitted pulse in Fig. 4, the use of a pendulum resulted in a transmitted signal. The transmitted signal directly gives engineering stress history through Eq. (3), where the cross-sectional area and Young's modulus of the bar. The incident and reflected signals give the dynamic strain history in the specimen through Eq. (4). 


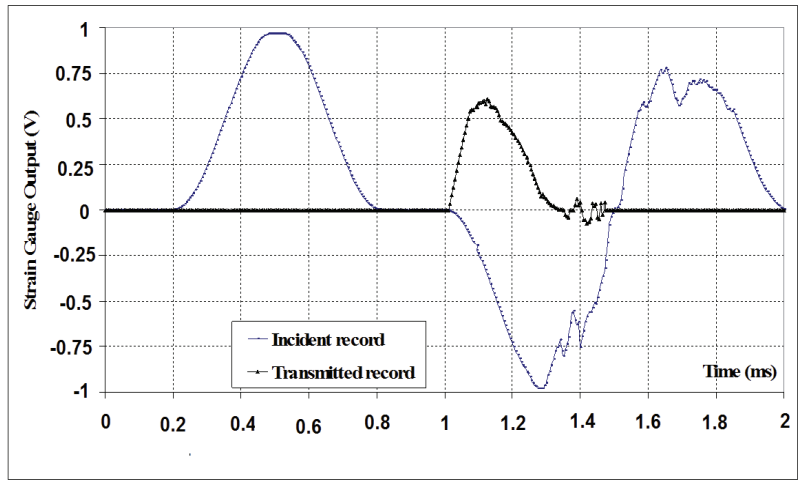

Fig. 4. The incident and the transmitted waves recorded by the stain gauges located on the bar and the sensing block under a dynamic tensile test on PPC7712

Figure 5 and 6, presents respectively the typical dynamic strain and the strain history for the specimen. The fact that the strain increases almost linearly with increasing time during the majority of the experiment indicates that a dynamic constant strain rate has been achieved. The slope is taken as the strain rate of the experiment.

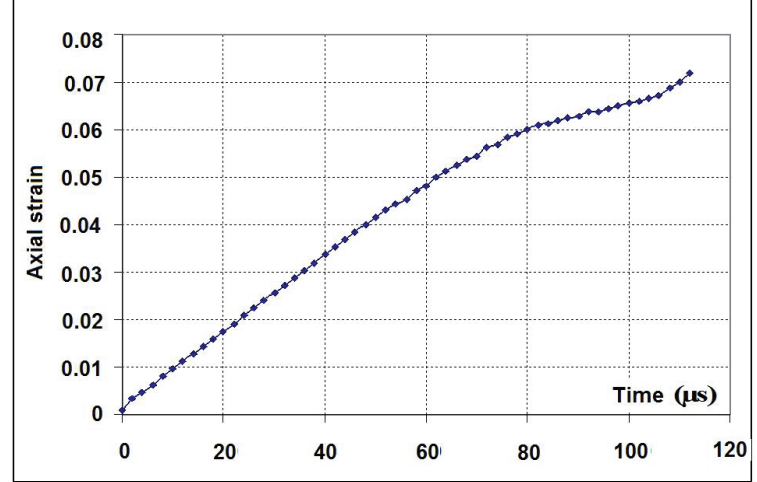

Fig. 5. The axial strain on the specimen versus time under a dynamic tensile test on PPC7712

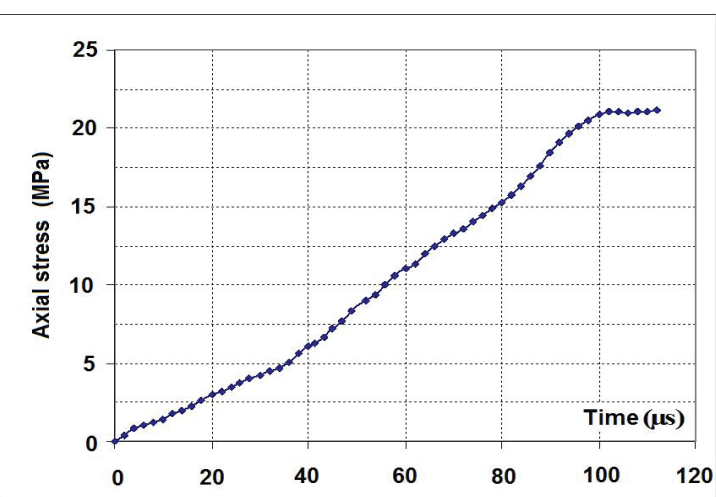

Fig. 6. The axial stress on the specimen versus time under a dynamic tensile test on PPC7712

Figure 7 summarizes the tensile stress-strain curves of PPC7712 over a strain-rate range of $100 \mathrm{~s}^{-1}$, $200 \mathrm{~s}^{-1}, 300 \mathrm{~s}^{-1}, 400 \mathrm{~s}^{-1}$ and $500 \mathrm{~s}^{-1}$, It should be noted that the stress-strain curves obtained from dynamic tensile machine experiments do not give reliable elastic modulus due to the transient stress state within the specimen during the early stages of the experiment. An inspection of Fig. 7 indicates that peak strength was reached during a dynamic test at a smaller strain compared with the quasi-static case. In addition, the results in Fig. 7 do not show clear strain-rate dependence of the material's peak tensile strength, which is approximately $25 \mathrm{MPa}$. The specimens fractured in a brittle manner during dynamic tensile loading. In contrast, during quasi-static tests, the specimens failed in a ductile manner with a necking process. The brittle-ductile transition is considered to be the strain-rate effect.

Qualitatively, if the polymer is still thermorheologically simple at such high strain rates encountered in the dynamic tensile machine range, and time superposition still applies, a higher strain rate would correspond to a shorter loading time. Another possible cause for the brittleductile transition is the short gage length of the specimen. The end constraint, in the form of the hydrostatic component of the stress tensor, to the gage section becomes more important as the length of the gage section decreases. The fact that more than one fracture surface exists, as shown in specimen, indicates that dynamic stress equilibrium has been reached before fracture events took place. However, the fracture occurred near the fillets despite a series of changes in the fillet radius in a series of trial experiments. This fact indicts that stress concentration near the fillets 


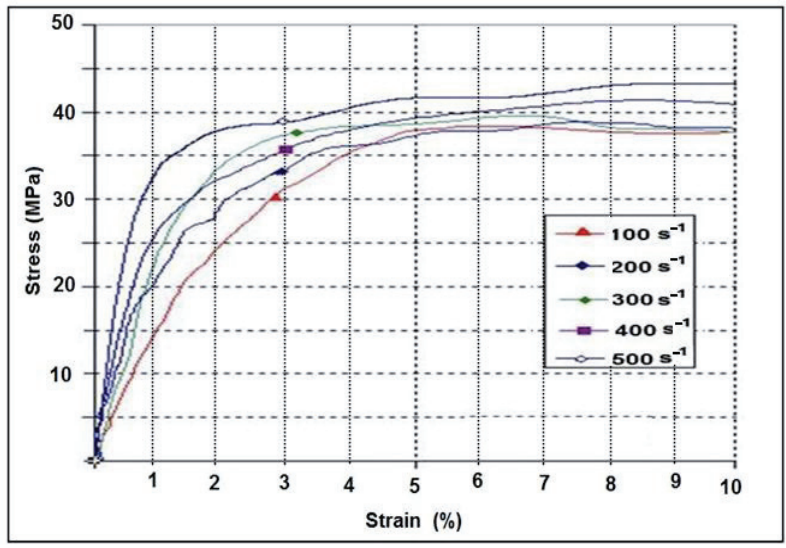

Fig. 7. Dynamic tensile test: stress-strain curves of PPC7712 for various strain rates

played an important role in the dynamic fracture of the short specimen, which may have shadowed the strain-rate effects on the tensile failure strength. As will be shown in the following discussion, the tensile experimental results exhibit much more apparent strain-rate effects on the peak strength of the same material.

Figure 8 presents two specimens after rupture for quasi-static and dynamic tests. From This figure, we can get an idea for the PPC7712 behaviour. It is shown that the final length of the specimen is more important for the quasi-static test Fig. 8 (a). That means the material has a ductile behaviour in the quasi-static test however it has a brittle behaviour in the dynamic test.

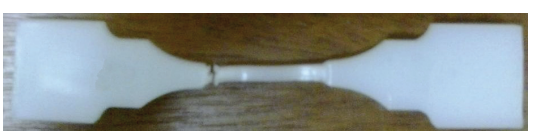

(a)

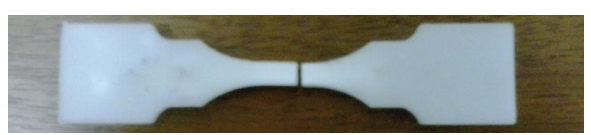

(b)

Fig. 8. The specimens after rupture (a) quasi-static test, (b) dynamic test

\section{Conclusion}

The quasi-static and dynamic stress-strain behaviour of a PPC7712 polypropylene has been determined under uniaxial tension loading conditions. To determine the dynamic tensile behaviour of low-strength, low-impedance of a PPC7712 polypropylene, a dynamic tensile machine has been developed to capture the low transmitted signal. A pulse-shaping technique was used to ensure a homogeneous deformation at a constant strain rate during experiment, either in tension or in compression.

For quasi-static case, the mechanical behaviour of this material is investigated at $0.8 \mathrm{~s}^{-1}$ strain rate. It is found the PPC7712 polypropylene has elasto-plastic behaviour and the Young's modulus can be determined. The specimen is failed in a ductile manner. It is noted that the dynamic stressstrain behaviour under tension differ from the dynamic compressive response for the quasi-static loading conditions. With similar quasi-static strength in both tension and compression states of the testing material, the dynamic compressive strength is higher than the quasi-static counterpart is.

For the dynamic case, a strain-rate range of $100 \mathrm{~s}^{-1}, 200 \mathrm{~s}^{-1}, 300 \mathrm{~s}^{-1}, 400 \mathrm{~s}^{-1}$ and $500 \mathrm{~s}^{-1}$ are considered in the experimental tests. The Young's modulus was found to increase with the strain rate. The experimental results show that, for the PPC7712 materials investigated, the dynamic stress-strain behaviour under tension significantly differ from the dynamic compressive response. Specimens are failed in a brittle manner under dynamic tension. Therefore, dynamic compressive results are not indicative for dynamic tensile failures. It is a challenge to design a valid dynamic tensile experiment for a polymeric material. Hence, the low wave speeds in the material significantly restrict the specimen length. 


\section{References}

[1] Hamoda, A. M. S., Hashmi, M. S. J., Testing of composite material at high rates of strain: advances and challenges, Journal of Material Processing and Technology, Vol. 77, pp. 327336, 1998.

[2] Kolsky, H., An Investigation of the Mechanical Properties of Materials at Very High Rates of Loading, Proc. Roy. Soc., B62, pp. 676-700, London 1949.

[3] Q.M. Li, Y.B. Lu, H. Meng., Further investigation on the dynamic compressive strength enhancement of concrete-like materials based on split Hopkinson pressure bar tests, International Journal of Impact Engineering, Vol. 36, Is. 12, pp. 1336-1345, 2009.

[4] Chou, S. C., Robertson, K. D., Rainey, J. H., The effect of strain rate and heat developed during deformation on the stress-strain curve of plastics, Experimental Mechanics, Vol. 13, pp. 422-432, 1973.

[5] Walley, S. M., Field, J. E., Pope, P. H., Safford, N. A., A study of the rapid deformation behaviour of a range of polymers, Philosophical Transactions of the Royal Society of London, 328, pp. 1-33, 1989.

[6] Dioh, N. N., Leevers, P. S., Williams, J. G., Thickness effects in split Hopkinson pressure bar tests, Polymer, Vol. 34, pp. 4230-4234, 1993.

[7] Khlif, M., Masmoudi, N., Bradai, C., Grolleau, V., Rio, G., Development of a new testing method for polymer materials at high strain rate, Journal of Theoretical and Applied Mechanics, Vol. 38, No. 4, pp. 67-82, 2008.

[8] Tanimura, S., Evaluation of Accuracy in Measurement of Dynamic Load by Using Load Sensing Block Method, Proceedings of 4th International Symposium on Impact Engineering ISIE4, pp. 77-82, Kumamoto, Japan 2001.

[9] Grolleau, V., Guines, D., Umiastowski, S., Ragneau, E., Rio, G., Optimisation de dispositifs d'essais dynamiques sur machine d'impact et presse hydraulique, MECAMAT, Aussois, 2005.

[10] Chen, W., Lu, F., Zhou, B., A quartz-crystal-embedded split Hopkinson pressure bar for soft materials, Experimental Mechanics, 40, pp. 1-6, 2000.

[11] Frew, D. J., Forrestal, M. J., Chen, W., A split Hopkinson bar technique to determine compressive stress-strain data for rock materials, Experimental Mechanics, Vol. 41, pp. 40-46, 2001.

[12] Zrida, M., Laurent, H., Rio, G., Pimbert, S., Grolleau, V., Masmoudi, N., Bradai, C., Experimental and numerical study of polypropylene behavior using an hyper-visco-hysteresis constitutive law, Computational Materials Science 45 (2), pp. 516-527, 2009.

[13] Krevelen, S. D. W. V., Properties of Polymer, Elsevier, 2009.

[14] Krawczak, P., Essais mécanique des plastiques, Techniques de l'Ingénieur, AM, 3, pp. 510, 1997. 